\title{
Article \\ Discussion on the Stiffness of the Drive Chain in the Legs of Biped Robots
}

\author{
Ruilong Du ${ }^{1,+} \mathbb{C}$, Sumian Song ${ }^{1, *+}{ }^{+}$, Haihui Yuan ${ }^{1, *}$, Daming $\mathrm{Nie}^{1}$ and Jason $\mathrm{Gu}^{2}$ \\ 1 The Intelligent Robot Research Center, Zhejiang Lab, Hangzhou 311121, China; durl@zhejianglab.com (R.D.); \\ niedaming@zhejianglab.com (D.N.) \\ 2 Department of Electrical Engineering, Dalhousie University, Halifax, NS B3H4R2, Canada; jason.gu@dal.ca \\ * Correspondence: songsm1002@163.com (S.S.); hh_yuan@zju.edu.cn (H.Y.) \\ + These authors contributed equally to this work.
}

Citation: Du, R.; Song, S.; Yuan, H.; Nie, D.; Gu, J. Discussion on the

Stiffness of the Drive Chain in the

Legs of Biped Robots. Actuators 2022,

11, 79. https://doi.org/10.3390/

act11030079

Academic Editor: Gianluca Palli

Received: 21 January 2022

Accepted: 1 March 2022

Published: 4 March 2022

Publisher's Note: MDPI stays neutral with regard to jurisdictional claims in published maps and institutional affiliations.

Copyright: () 2022 by the authors. Licensee MDPI, Basel, Switzerland. This article is an open access article distributed under the terms and conditions of the Creative Commons Attribution (CC BY) license (https:// creativecommons.org/licenses/by/ $4.0 /)$.

\begin{abstract}
Biped robots' locomotion is realized by driving the joint motion via a drive chain. Therefore, the stiffness of the drive chain is an important factor that affects the drive performance and can influence the locomotion behavior of the biped robot. This work focused on the influence of the stiffness of the leg's drive chain using a mass-spring model based on the biped robot AIRO built in Zhejiang Lab. Methods for determination of the parameters in the proposed model were presented, including the use of ANSYS Workbench to determine the stiffness parameters and the determination of the inertia parameters by dynamic modelling of the biped robot. Simulation results show that special attention should be paid to the stiffness of the drive train of the leg when designing a biped robot to ensure the walking capability of the robot. Using the model proposed in this work, relations between the executed accuracy of the joint trajectories and the stiffness can be analyzed; after that, the stiffness parameters can be optimized. In addition, simulation results also showed that attention should be paid to manufacturing tolerances to ensure the symmetry of the legs of the bipedal robot in order to reduce the vibration of the robot body. Experiments were conducted on AIRO for validating the proposed model and the simulation analysis.
\end{abstract}

Keywords: biped robot; drive chain; stiffness; mass-spring model; dynamic modelling of the biped

\section{Introduction}

Biped robots have attracted a lot of attention in recent years for their unique advantage of human-like structure, which allows them to easily work in a human-built environment [1]. Up to now, a multitude of biped robot designs have been developed, such as Atlas, developed by Boston Dynamics [2], Cassie, developed by the Oregon State University [3], Asimo, developed by the Honda company [4], and others [5,6]. Though developed by different research groups, what these biped robots have in common is the anthropomorphic leg structure, which is comprised of mechanical components, drive systems and transmission mechanisms, such as the thigh, the electric motor and the linkage, mechanism,

Typically, biped robots are driven by electric motors [7,8], hydraulics $[9,10]$ or pneumatics [11,12]. With respect to the publications of biped robots, most of them address the issues of bionic structure designs, gait panning, and control strategies [13-15]. In these publications, the biped robots are usually taken as rigid bodies, which is not feasible in the real design, regardless of the driving approach. Hence, it is worth noting that the biped robots, in particular their legs, should be taken as flexible parts. Elastic deformation would occur when subjected to external forces during the biped robot's locomotion process; consequently, the actual execution of the joint trajectory might deviate from the expected trajectory. For example, due to the elastic deformation of the drive chain of the knee joint motion, the execution angle position of the knee joint may deviate from its gait planning. 
Therefore, the stiffness of the drive chain would have an influence on the locomotion behavior of biped robots and is worth discussion.

However, few publications could be found on discussing the stiffness of the leg's drive chain. Carbone et al. analyzed the static characteristics of the biped robot Wabian-RIV induced by stiffness. In their works, the robot was regarded as springs and the stiffness parameters were determined by measuring the displacement with respect to a certain static wrench being applied to the robot [16-18]. However, the dynamic behavior of the robot was not accounted for. Kwon et al. analyzed the dynamic stiffness of mechanical components of the biped robot Mahru III for mass reduction using CAE methods [19,20]. However, only mechanical components were analyzed and the drive chain system was not accounted for. Lohmeier performed an elasto-dynamic analysis of the drive mechanisms of the biped robot, Lola utilizing two-mass systems as an equivalent model [21-23]. However, the stiffness of the leg's stiffness was not accounted for. Xiong et al. discussed the leg stiffness based on the motion of the spring joints and the motor joints. However, Xiong's work mainly focused on jumping control of the biped, while the stiffness of the drive chain was not discussed [24,25]. Moreover, it is worth noting that, in the above works, the legs of the biped robots were taken as two symmetrical legs. However, due to manufacturing errors, the two legs were not completely symmetrical; this would do harm to the locomotion behavior of the biped robot.

Research could be found on addressing the stiffness concerning other robotic systems, such as industrial robots, the lower limb exosuits, et al. [26,27]. Luca et al. analyzed the robot manipulator's performance, taking the mechanical flexibility in to consideration, focusing on the stiffness and damping properties [28]. Klimchik et al. proposed a stiffness model for a serial robot, paying particular attention to the elastostatic parameters identification and calibration of the robot [29]. Pashkevich et al. presented a methodology to enhance the stiffness analysis of serial manipulators, taking into account the loading influence on the manipulator configuration [30]. Kim et al. analyzed the stiffness and optimized the design of an under-actuated tendon-driven robot [31]. Geeroms et al. designed and analyzed a prosthetic knee joint actuator with a lockable parallel spring [32]. However, in these works, the industrial robots were connected to fixed bases and lower limb exosuits usually played the role of assisting and interacting with humans; the biped robots are mobile robots that usually move on their own, with two legs that each take turns as the base during locomotion. Under this circumstance, the drive chain of the leg is of particular interest and discussion.

This work used the biped robot AIRO, shown in Figure 1, as a platform. AIRO was designed and built by us in Zhejiang Lab. In this work, we conclude some lessons learned from designing and testing the biped robot AIRO and elucidate the effects of the stiffness of the leg's drive chain on the locomotion behavior in biped robots using mathematical models. The model was experimentally validated using AIRO and the parameters of the leg's drive chain of AIRO were further optimized.

The main contribution of this work are as follows: (1) a mass-spring model was established that allows analysis of the influence of the stiffness of the drive chain of the leg; (2) methods for determination of the parameters of the stiffness and the moment of inertia were introduced, including using ANSYS Workbench and the dynamic modelling of the biped robot; (3) guidance was given for the design of the biped robot's legs, including the leg's drive chain and the symmetrical design of the two legs.

The rest of this work is organized as follows. Section 2 describes the structural design of the biped robot AIRO, especially its leg structure and the drive chain. Section 3 brings the mathematical model for analyzing the stiffness of the drive chain. Section 4 describes the simulation results and related discussions on the influence of the stiffness. Section 5 shows the experiment setup and related experimental results. After that, Section 6 presents the conclusions and the future work. 


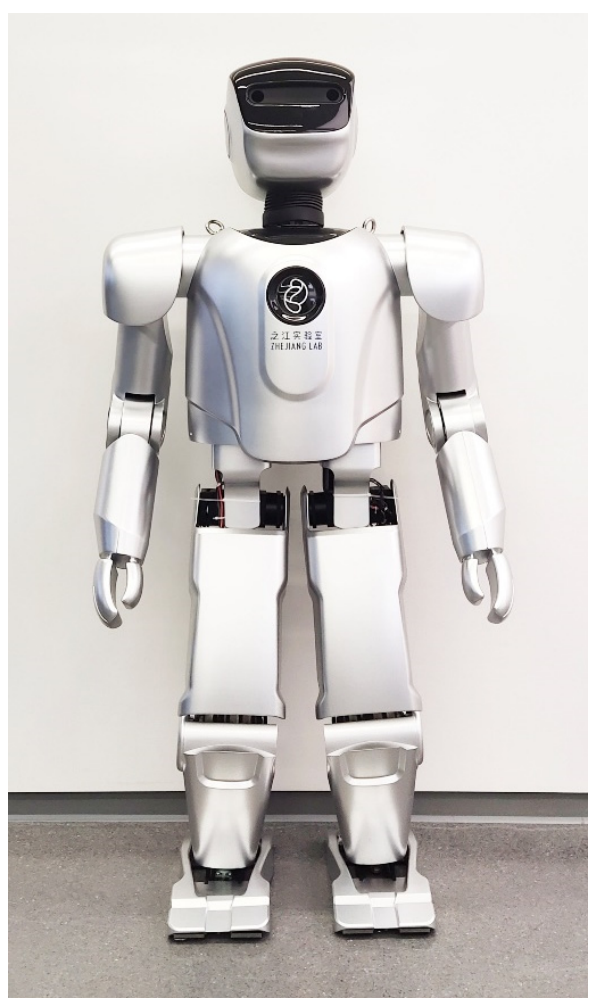

Figure 1. Biped robot AIRO built in Zhejiang Lab.

\section{Description of the Biped Robot AIRO}

Figure 2 presents the configuration of AIRO, a biped robot built in Zhejiang Lab. The robot was approximately $30 \mathrm{~kg}$ in weight, $1.4 \mathrm{~m}$ in height and $0.625 \mathrm{~m}$ in width. AIRO's leg weighed $8 \mathrm{~kg}$ and its length was $600 \mathrm{~mm}$, including $300 \mathrm{~mm}$ for the thigh and $300 \mathrm{~mm}$ for the shank. AIRO had 20 degrees of freedom (DOFs), including 2 for the head, 3 for each arm, and 6 for each leg. Regarding the leg, it consisted of three working parts: the hip joint with $3 \mathrm{DOFs}$, the knee joint with $1 \mathrm{DOF}$ and the ankle joint with 2 DOFs. It was expected that AIRO could achieve omnidirectional walking with a maximum step size of $488 \mathrm{~mm}$ for forward walking and $328 \mathrm{~mm}$ for lateral walking.
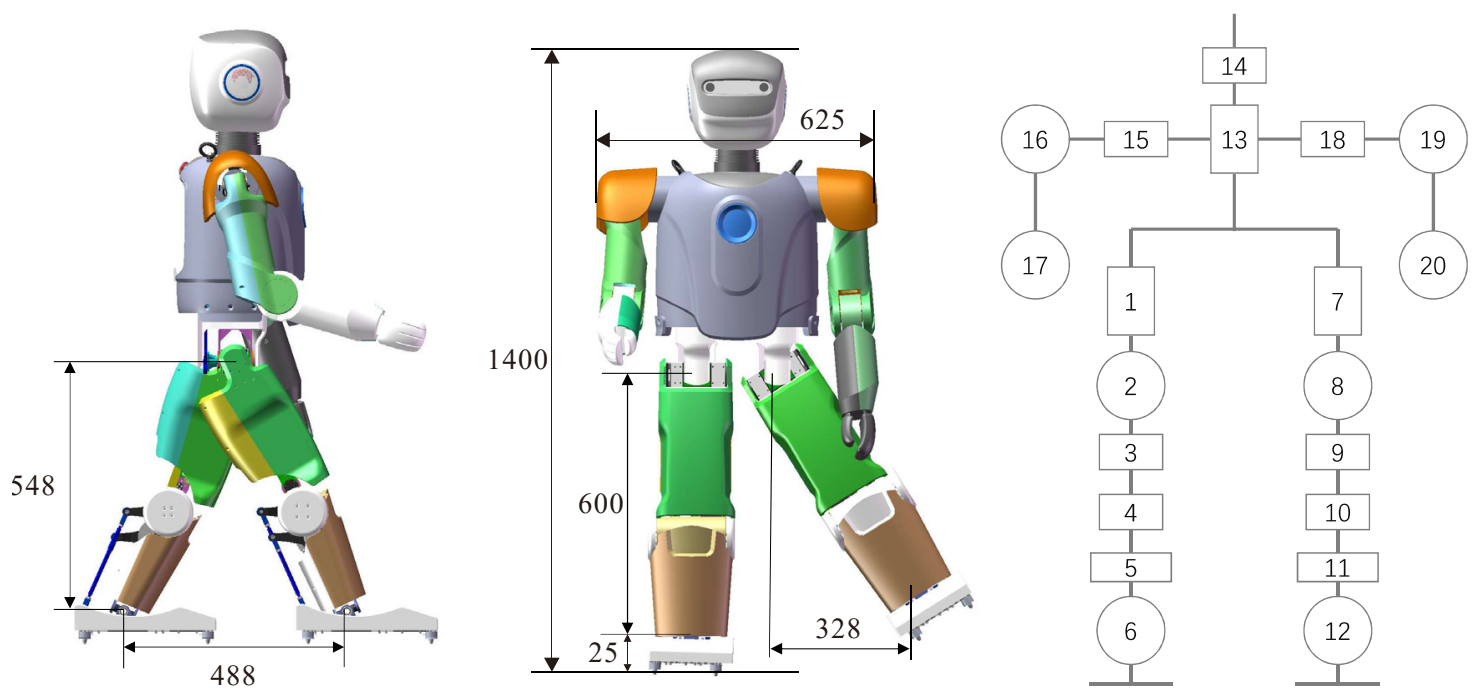

Figure 2. Configuration of the biped robot AIRO built in Zhejiang Lab. 
The main design concept for AIRO, especially the leg structure, is based on the inverted pendulum model, which is characterized by a mass mainly concentrated above the leg and by the leg being lightweight. To satisfy this, as shown in Figure 3a, the motors and the joint motions they drive are connected via linkages such that the motors can be placed closer to the hip, reducing the weight of the distal end of the leg. Specifically, the motor for driving motion of the knee is placed in the thigh; the motors for driving the motion of the ankle are placed in the shank.

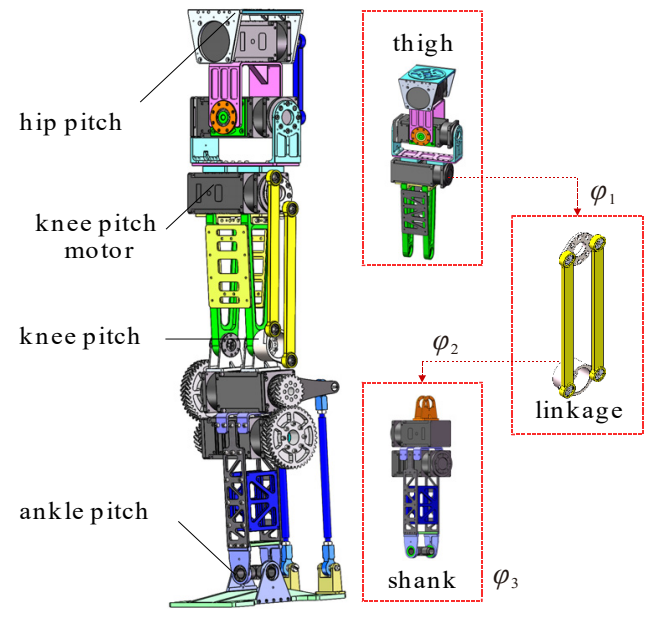

(a)

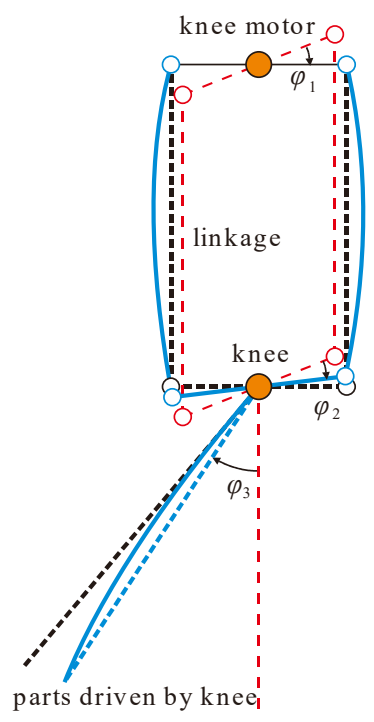

(b)

Figure 3. Leg structure of the biped robot AIRO: (a) Drive chain of the knee pitch motion; (b) Schematic representation of the knee pitch motion.

AIRO is expected to be able to travel with only position control of the motor, without measuring and feeding back the actual trajectory of the joint. Specifically, the motors with encoders integrated within would receive commands from the walking gait generator and then execute the corresponding rotation motion. For a simpler leg structure and a lower cost, no extra encoders were added on the joints. By rotating the motors, the joints are driven to rotate an angle via the linkages, i.e., the drive chain. The motors are seen to rotate the same angles with the commands generated by the walking gaits; it is hoped that the angles at which the joints rotate should be the same as the angles at which the motors rotate.

However, during the period of the preliminary testing of the robot walking, two unfavorable walking phenomena of AIRO could be observed: (1) AIRO was observed to be unable to lift his legs into the air and then touch the ground alternately; (2) A relatively large upper body vibration could be observed; the upper body vibrated with different magnitudes in different directions, despite the symmetrical design of the two legs.

As mentioned above, we firstly examined the feedback data of the motors, and confirmed that the output trajectories of the motors matched well with those produced by the gait planning, proving that the output speed and torque of the motor were adequate.

After a thorough test of the robot walking, it was found out that the knee pitch motion trajectory deviated from the trajectory generated by the walking gaits, leading to the two unfavorable walking phenomena described above.

The reason behind these phenomena lies in the stiffness of the leg's drive chain. To be specific, as shown in Figure 3a, the knee pitch motion was driven via a linkage between the hip pitch motor and the knee joint, where a parallelogram was formed between the output of the motor and the knee pitch joint. As shown in Figure 3b, the red lines denote the initial position of linkages. If the parallelogram was a rigid body, the rotation angle of the knee pitch motion would be the same as the output of the motor, as shown by the black lines, 
which can be expressed as $\varphi_{1}=\varphi_{2}$. However, due to the elastic deflection of the linkages, the rotation angle of the knee pitch motion could not catch up with the output of the motor, as shown by the blue lines, which meant that $\varphi_{2}$ was no longer equal to $\varphi_{1}$.

In addition to this, for the biped robot's walking, some parts would be driven by the knee pitch motion, as shown in Figure 3a for the shank. If those parts were rigid bodies, they would be driven by the same rotation angle by the knee pitch motion, as shown by the black line in Figure 3b, which can be expressed as $\varphi_{2}=\varphi_{3}$. However, these parts would also undergo elastic deformation, leading to a different value of $\varphi_{3}$ to that of $\varphi_{2}$, as shown by the blue lines.

Hence, judging from the analysis above, by not measuring and feeding back the actual trajectory of the joint during walking, the actual rotation angle of the parts driven by the knee pitch motion $\varphi_{3}$ would deviate from the angle $\varphi_{1}$ generated by gait planning, which would inevitably lead to a negative influence on the locomotion behavior of the robot. Manufacturing and assembly errors lead to differences in the stiffness of the drive chain in the two legs, which, in turn, caused the upper body of AIRO to vibrate in different directions at different degrees.

A further discussion on the relations between the angles of $\varphi_{1}, \varphi_{2}$ and $\varphi_{3}$ needs to be addressed to provide guidance for optimizing the structure of the leg and to improve the walking performance.

\section{Mathematical Modelling for the Knee Motion}

\subsection{Spring-Mass Models for the Knee Motion}

As analyzed above, the deviation of the angles of $\varphi_{1}, \varphi_{2}$ and $\varphi_{3} \mathrm{n}$ was mainly caused by the elastic deflection of the leg structure. From a mechanical point of view, the deviation caused by elastic deformation can be considered as an open kinematic chain composed of rigid bodies and springs, as shown in Figure 4. Therefore, the angles of $\varphi_{1}, \varphi_{2}$ and $\varphi_{3}$ could be represented using the mass-spring model; the dynamic model for the rigid bodies rotation angle could be expressed as Equation (1).

$$
\left[\begin{array}{ccc}
I_{1} & 0 & 0 \\
0 & I_{2} & 0 \\
0 & 0 & I_{3}
\end{array}\right]\left[\begin{array}{l}
\ddot{\varphi}_{1} \\
\ddot{\varphi}_{2} \\
\ddot{\varphi}_{3}
\end{array}\right]+\left[\begin{array}{ccc}
k_{1} & -k_{1} & 0 \\
-k_{1} & k_{1}+k_{2} & -k_{2} \\
0 & -k_{2} & k_{2}
\end{array}\right]\left[\begin{array}{l}
\varphi_{1} \\
\varphi_{2} \\
\varphi_{3}
\end{array}\right]=\left[\begin{array}{c}
T_{1} \\
0 \\
T_{3}
\end{array}\right]
$$

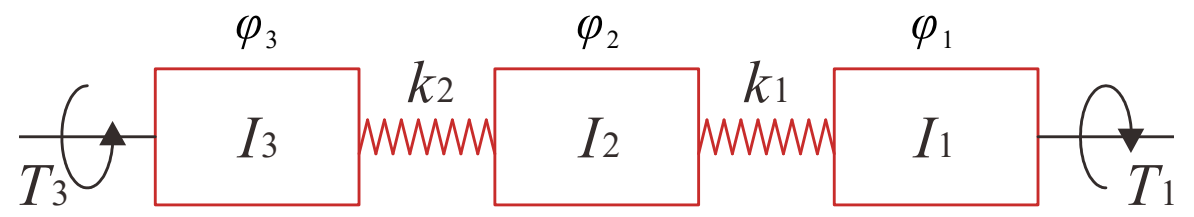

Figure 4. Schematic of the drive chain for the knee pitch motion using a mass-spring model.

The term $T_{1}$ denotes the motor's output torque acting on the proximal end of the linkages for driving the knee pitch motion; the term $T_{3}$ denotes the torque for driving the parts of the robot via the knee pitch motion.

The terms $\varphi_{1}$ denotes the rotation angle of the motor, which is also the rotation angle of the proximal end of the linkages; the term $\varphi_{2}$ denotes the rotation angle of the knee joint, which is also the rotation angle of the distal end of the linkages; the term $\varphi_{3}$ denotes the rotation angle of the parts driven by the knee pitch motion.

The term $I_{1}$ denotes the moment of inertia of the knee pitch motor, the first rigid body; the term $I_{2}$ denotes the moment of inertia of the linkages, the second rigid body; the term $I_{3}$ denotes the moment of inertia of the parts driven by the knee pitch motion, the third rigid body.

The term $k_{1}$ denotes the stiffness of the linkages, the first spring; the term $k_{2}$ denotes the stiffness of the parts driven by the knee pitch motion, the second spring. 
It is worth noting that the parts driven by the knee pitch motion refer to a dynamically varying structure. As presented in Figure 5, during the biped locomotion, the leg switched between the swing phase and the stance phase periodically. It was assumed that there was no double-stance phase, namely, when one leg touched the ground, the other leg would leave the ground at the same moment. It was also assumed that there was no slippage in the stance phase. When the leg switched from one phase to the other, the parts driven by the knee pitch motion would also be different. To be specific, with respect to the swing leg, the knee pitch motion drove the shank forward in the air, which was characterized by a relatively higher moving speed and a low load inertial. With respect to the standing leg, the knee pitch motion must support the robot itself and must drive it forward, which was characterized by a relatively lower moving speed and a high load inertial.

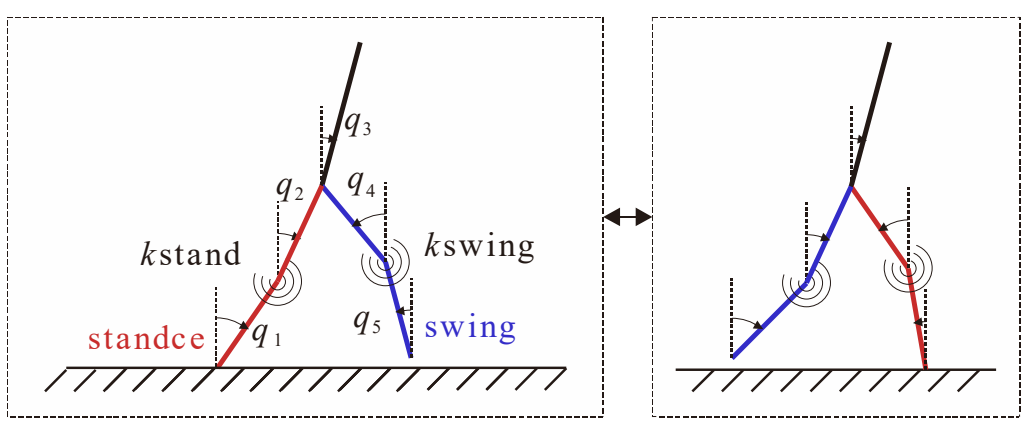

Figure 5. Structure-varying topology of the biped robot during walking.

\subsection{Determination of Parameters}

As stated above, the term $\varphi_{1}$ denotes the rotation angle of the knee pitch motor, consistent with that from the gait planning. For evaluating the difference between the terms $\varphi_{1}, \varphi_{2}$ and $\varphi_{3}$, other parameters in Equation (1) need to be determined first.

The terms $I_{1}$ and $I_{2}$ can be calculated using the definition of the moment of inertia. As concerns the term $I_{3}$, it varies as the robot's topology varies. Hence, term $I_{3}$ needs to be estimated via the dynamic modelling of the robot. As presented in Figure 5, the dynamic model for the biped robot can be expressed as Equation (2).

$$
M \cdot \ddot{q}+C \cdot \dot{q}+G=B \cdot u
$$

where $M$ denotes the joint-space inertia matrix relating to the joint angular accelerations $\ddot{q}$. $C$ denotes the Coriolis matrix relating to the joint angular velocities $\dot{q}$. $G$ denotes the gravity matrix.

Noting that the term $\ddot{q}$ is a vector composed of the angular accelerations of all joints, Equation (2) can be re-written as Equation (3).

$$
\sum_{i=1}^{n}\left(\left(\sum_{j=1}^{n} M(j, i)\right) \cdot \ddot{q}_{i}\right)+C \cdot \dot{q}+G=B \cdot u
$$

Hence, for the knee pitch motion $q_{k}$, Equation (3) can be further written as Equation (4).

$$
\left(\sum_{j=1}^{n} M(j, k)\right) \cdot \ddot{q}_{k}=B \cdot u-C \cdot \dot{q}-G-\sum_{i=1}^{k}\left(\left(\sum_{j=1}^{n} M(j, i)\right) \cdot \ddot{q}_{i}\right)-\sum_{i=k+1}^{n}\left(\left(\sum_{j=1}^{n} M(j, i)\right) \cdot \ddot{q}_{i}\right)
$$

Judging for Equations (1) and (4), for knee pitch motion $q_{k}$, the term $I_{3}$ can be evaluated as Equation (5).

$$
I_{3}=\left(\sum_{j=1}^{n} M(j, k)\right)
$$


The term $T_{3}$ can be obtained from Equation (4) as well, as shown in Equation (6).

$$
T_{3}=B \cdot u-C \cdot \dot{q}-G-\sum_{i=1}^{k}\left(\left(\sum_{j=1}^{n} M(j, i)\right) \cdot \ddot{q}_{i}\right)-\sum_{i=k+1}^{n}\left(\left(\sum_{j=1}^{n} M(j, i)\right) \cdot \ddot{q}_{i}\right)
$$

The term $k_{1}$ represents the stiffness of the linkages, which can be defined as the ratio of the torque acting on the linkages to the related deformation of the linkages. The deformation can be determined using ANSYS Workbench. As shown in Figure 6a, the linkages were composed of an output ring connected to the motor's output, a connector connected to the knee pitch joint, and two linkage bars for transmission, all of which were made of 7075 aluminum. The length of the linkages was $200 \mathrm{~mm}$; the width was $20 \mathrm{~mm}$; the thickness was $5 \mathrm{~mm}$. The two ends of the linkages were glued tightly to the output ring and the connector, respectively. The transmission ratio was 1 for the output ring; the connector had the same diameter $(2 r)$.

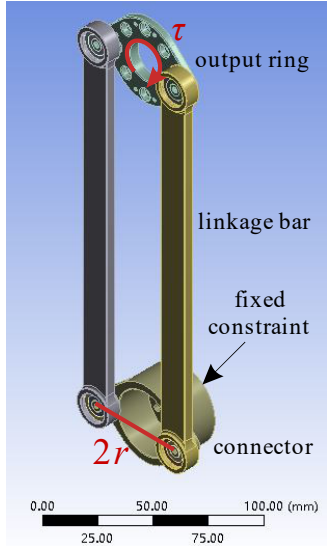

(a)

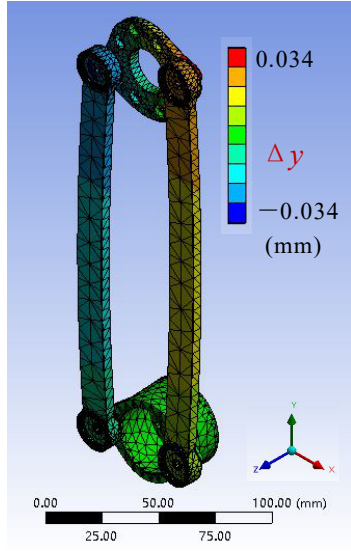

(b)

Figure 6. (a) structure presentation of the linkages; (b) Deformation of the linkages with respect to a certain torque.

For evaluating the deformation with respect to a certain torque, a fixed constraint was applied to the connector and a $20 \mathrm{Nm}$ torque $\tau$ was applied to the output ring. As shown in Figure $6 \mathrm{~b}$, deformation $\Delta y$ in the $y$ axis direction could be obtained and the stiffness parameter $k_{1}$ of the linkages can be calculated as Equation (7).

$$
k_{1}=\frac{\tau}{\Delta y / r}=\frac{\tau \cdot r}{\Delta y}
$$

It is worth noting that $k_{1}$ varied as the output ring rotated, since the pressure angle between the output ring and the linkage bar varied. According to the gait planning, the range of the knee pitch motion was $40^{\circ}$, and the rotation range of the linkages lied between $10^{\circ}$ and $50^{\circ}$. As shown in Figure 7a, the deformation of $\varphi=50^{\circ}$ was approximately twice that of $\varphi=10^{\circ}$, indicating that the stiffness of $\varphi=10^{\circ}$ was approximately twice that of $\varphi=50^{\circ}$. Considering the design and installation of the linkages, two types of the output ring's installation could be realized: type a for rotation from $10^{\circ}$ to $50^{\circ}\left(k_{1}\right)$ and type b for rotation from $50^{\circ}$ to $10^{\circ}\left(k_{1^{\prime}}\right)$. Figure $7 \mathrm{~b}$ depicts the stiffness of the linkages regarding the two different installation types $\left(k_{1}\right.$ and $\left.k_{1^{\prime}}\right)$; the stiffness data evaluated by ANSYS could be further interpolated using polynomial curves. 


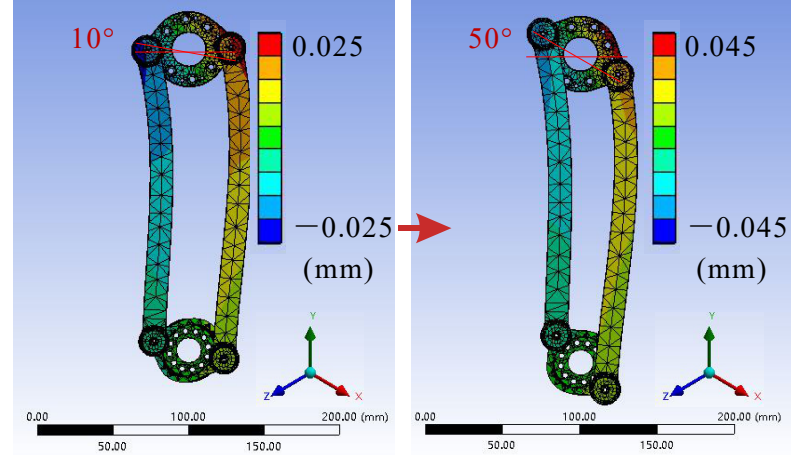

(a)

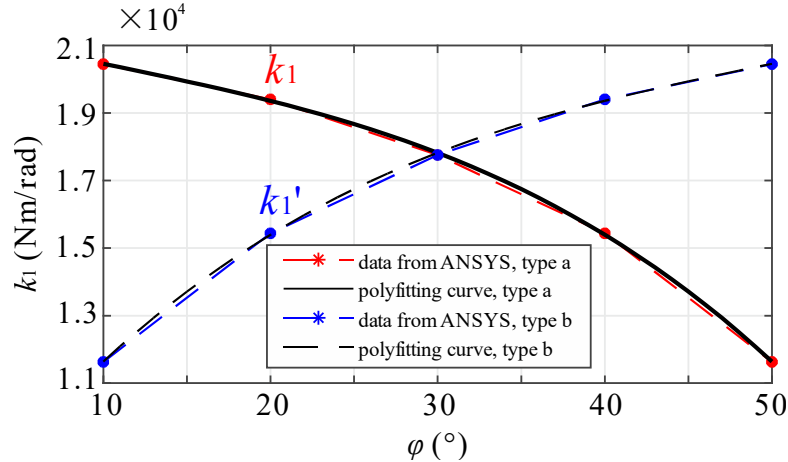

(b)

Figure 7. (a) Two types of installation of the linkages; (b) Stiffness of the linkages regarding different installation types.

The term $k_{2}$ represents the stiffness of the parts driven by the knee pitch motion, which can be taken as a cantilever beam and evaluated using ANSYS Workbench. Two different types of cantilever beams are accounted for, depending on the stance phase or the swing phase. Regarding the stance phase, the cantilever beam is formed by the thigh between the hip pitch joint and the knee pitch joint. Regarding the swing phase, the cantilever beam was formed by the shank between the knee pitch joint and the ankle pitch joint. Figure 8 shows the cantilever beam formed by the thigh for the stance phase. For the ANSYS evaluation, a fixed constraint was applied to the knee pitch joint, and a force of $100 \mathrm{~N}$ in the pitch direction, which is of the same order of magnitude as the weight of the leg $(8 \mathrm{~kg})$, was applied to the other end of the cantilever beam. Displacement in the pitch direction $\Delta x$ could be obtained and the stiffness parameter $k_{2}$ related to the leg structure could be evaluated in the same way as Equation (7).

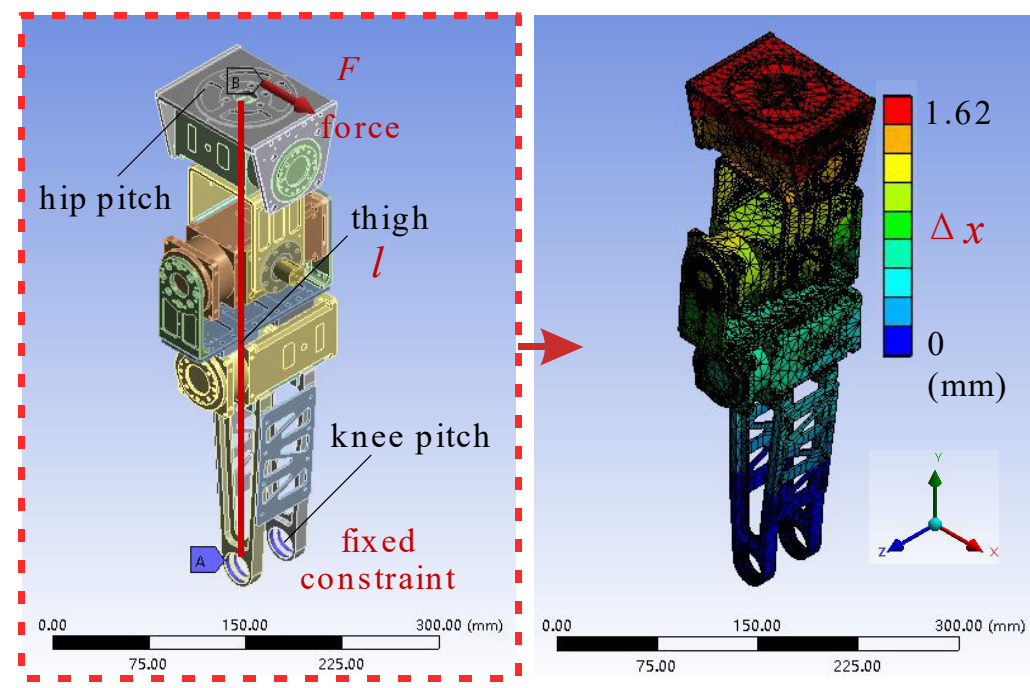

Figure 8. Stance phase, beam formed by thigh between hip and knee.

\subsection{Numerical Solution of the Mathematical Model}

In Equation (1), the term $\varphi_{1}$ is given by the gaiting planning, which is expressed in a sine curve, as shown in Equation (8). The terms $I_{1}, I_{2}$ and $k_{2}$ were evaluated in the last subsection; the term $k_{1}$ is a polynomial equation defined by $\varphi_{1}$ judging from Figure 7; the terms $I_{3}$ and $T_{3}$ are equations defined by $\varphi_{3}$ judging from Equations (5) and (6). Hence, Equation (1) is a set of $2^{\text {nd }}$ order ordinary differential equations composed of three equations 
with three unknown parameters $\varphi_{2}, \varphi_{3}$ and $T_{1}$, which can be numerically solved using the 4 th order Runge-Kutta method, converging to $1 \times 10^{-6}$.

$$
\varphi_{1}=-a_{x} \cos (2 \pi f t)+a_{x}
$$

where $f$ denotes the step frequency; $a_{\mathrm{x}}$ denotes amplitude of the sine curve, which is also half of the range of $\varphi_{1}$.

\section{Simulation Results on the Stiffness}

\subsection{Performance of the Stance and Swing Phase}

For comparison of the values of $\varphi_{1}, \varphi_{2}$ and $\varphi_{3}$, the installation type a for the linkages was adopted $\left(k_{1}\right)$. The evaluated stiffness for the leg structure (thigh or shank) $k_{2}$ was approximately $10,000 \mathrm{Nm} / \mathrm{rad}$. The amplitude of the sine curve $a_{\mathrm{x}}$ in Equation (8) was $20^{\circ}$ and the step frequency $f$ was $2.0 \mathrm{~Hz}$.

Figure 9 depicts the comparison of the values of $\varphi_{1}, \varphi_{2}$ and $\varphi_{3}$ for the stance phase. As shown in Figure 9a, the curve of $\varphi_{1}$ (the blue solid line) was a sine curve ranging from 0 to $40^{\circ}$; the curves of $\varphi_{2}$ and $\varphi_{3}$ were sine-like curves whose maximum and minimum values exhibited a wave-like envelope (red dashed line). The value of $\varphi_{2}$ (red solid line) ranged from $-0.7^{\circ}$ to $41.2^{\circ}$; the value of $\varphi_{3}$ (black solid line) ranged from $-2.0^{\circ}$ to $42.8^{\circ}$. The wave envelop suggests that the joint's motion range changed with each step, which, in turn, induced vibration of the robot. A roughly $5 \%$ error in magnitude was induced by the elastic deformation of the linkages and a roughly $7 \%$ error in magnitude was induced by the elastic deformation of the parts driven by knee pitch motion.

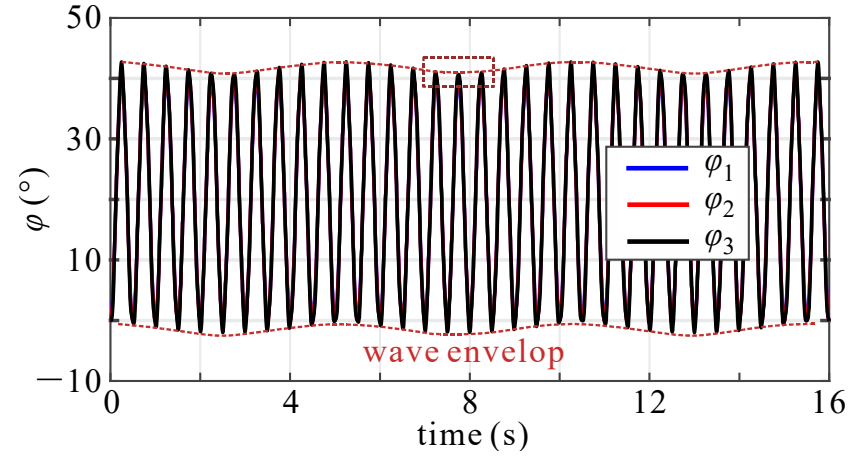

(a)

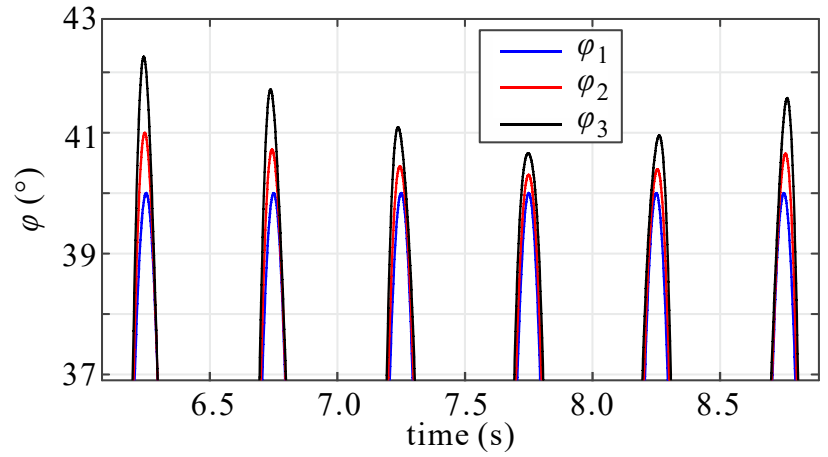

(b)

Figure 9. Stance phase, simulation results of $\varphi_{1}, \varphi_{2}$ and $\varphi_{3}$ : (a) Values of $\varphi_{1}, \varphi_{2}$ and $\varphi_{3}$ for the stance phase; (b) Enlarged view of the rectangular area in (a).

As shown in Figure 9b, the phase error between the three lines could be observed, especially the difference between the red line and the blue line. The maximum value of the red line could be observed sometimes in front of the red line and sometimes behind the red line. This is believed to do a greater harm since it is more difficult to reduce the error in phase than to reduce the error in amplitude.

An FFT of $\varphi_{1}, \varphi_{2}$ and $\varphi_{3}$ is depicted in Figure 10 in order to better understand the errors induced by the elastic deformation. Figure 10a depicts the FFT for the stance phase. As observed, there was only one peak at $2.0 \mathrm{~Hz}$ in the blue line since $\varphi_{1}$ is a sine curve with the frequency of $2.0 \mathrm{~Hz}$. As for the red line and the black line, greater amplitudes can be observed at $2.0 \mathrm{~Hz}$, suggesting a greater peak-to-peak value of $\varphi_{2}$ and $\varphi_{3}$, which corresponds to the errors in magnitude, as depicted in Figure 9a. Additionally, peaks could be seen at roughly $8.2 \mathrm{~Hz}$ for the red line and the black line, where the amplitude for the blue line was zero. It corresponded to the wave envelope and the errors in phase as depicted in Figure 9b. 


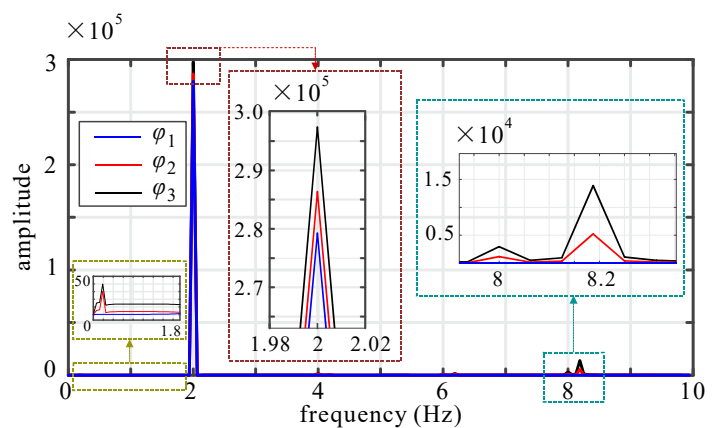

(a)

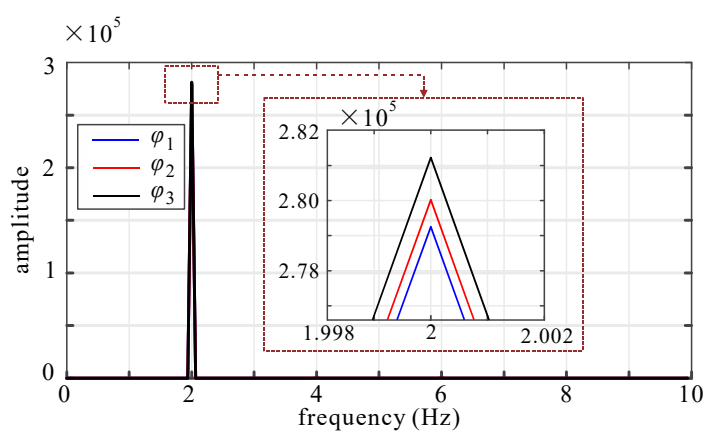

(b)

Figure 10. FFT of $\varphi_{1}, \varphi_{2}$ and $\varphi_{3}$ : (a) Stance phase; (b) Swing phase.

Figure 10b depicts the FFT for the swing phase. As observed, the peaks of the three lines at $2.0 \mathrm{~Hz}$ were very close, suggesting that there was no obvious wave envelops for $\varphi_{2}$ and $\varphi_{3}$. Apart from that, no significant values could be observed, besides $2.0 \mathrm{~Hz}$, suggesting that there were no obvious errors in phases for $\varphi_{2}$ and $\varphi_{3}$. Hence, this suggests that the stiffness for the swing phase was large enough and that the errors induced by the elastic deformation could be neglected.

\subsection{Influence of the Stiffness for Stance Phase}

In order to give guidance for optimizing the drive chain, further discussion is needed regarding the effect of stiffness in the stance phase. As discussed above, the error was induced by the elastic deformation of the linkage and the thigh structure between the knee pitch joint and the hip pitch joint. To reduce the elastic deformation, some mechanical components need to be bigger in size or strength (for example, using structural steel instead of aluminum), which would inevitably lead to a bigger size and a heavier weight of the leg; this is also detrimental to the biped robot. To solve this dilemma, different combinations of $k_{1}$ and $k_{2}$ were analyzed to find a good combination of $k_{1}$ and $k_{2}$.

Figure 11 depicts the FFT analysis of $\varphi_{3}$ for different combinations of $k_{1}$ and $k_{2}$ in the frequency domain above $2.0 \mathrm{~Hz}$. It can be observed that with the increase of $k_{1}$ and $k_{2}$, the frequency where peaks occur are getting bigger, and the amplitudes of the peaks are getting lower. This suggests that the changes in the wave shaping is smaller as the stiffness increases, indicating smaller induced errors. Additionally, the decrease rate of the amplitude becomes slower as $k_{1}$ and $k_{2}$ increase, suggesting that a good combination of $k_{1}$ and $k_{2}$ could be found considering the dilemma between the executed accuracy and the size and weight of the leg.

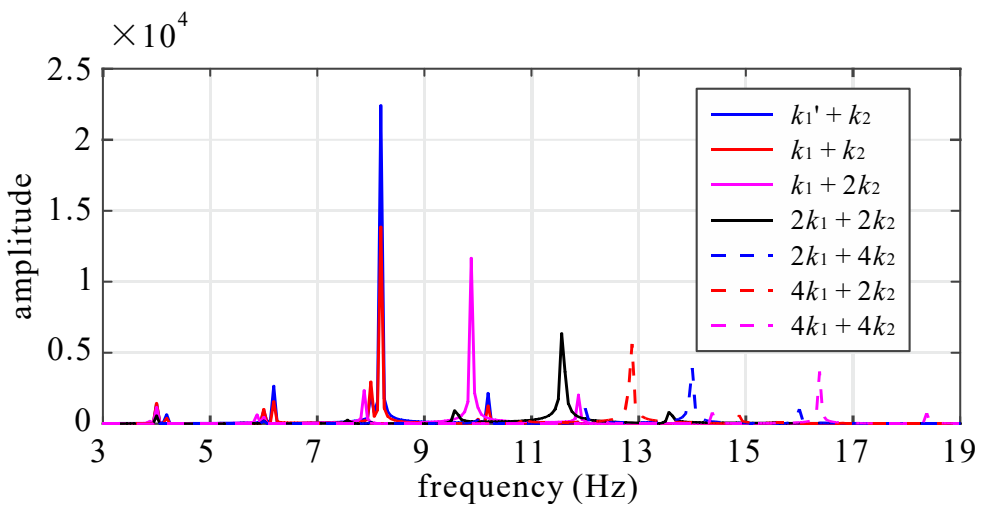

Figure 11. FFT Analysis of $\varphi_{3}$ regarding different stiffness.

Figure 12 depicts the errors induced by $k_{1}$ and $k_{2}$ and the total error regarding different combinations of $k_{1}$ and $k_{2}$. As $k_{1}$ and $k_{2}$ increased, the total error decreased, first rapidly 
and then slower and slower. At the combination of $2 k_{1}$ and $4 k_{2}$, a good compromise could be reached between the execution accuracy and the size and weight of the leg.

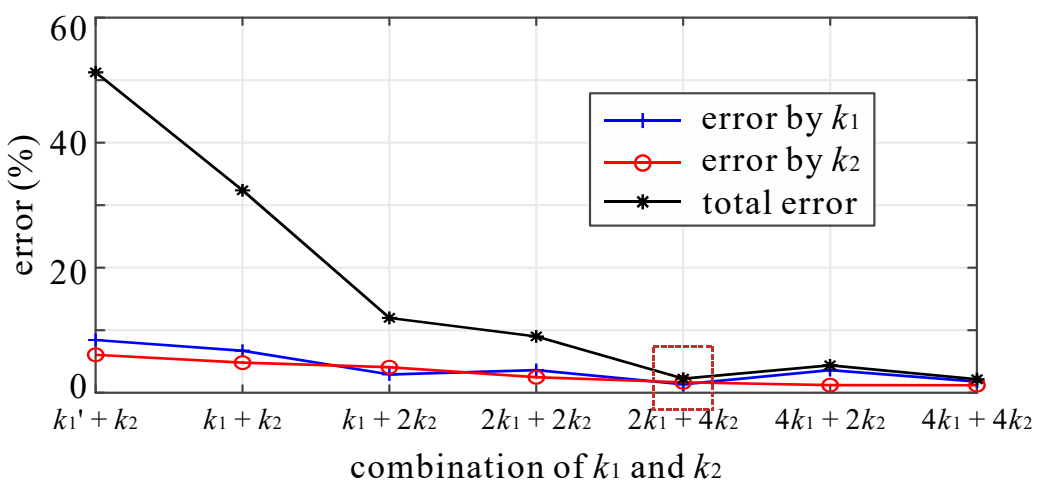

Figure 12. Induced errors regarding different stiffness.

\subsection{Influence of the Legs' Asymmetry}

To reduce robot manufacturing costs and make assembling easier, at the start, AIRO's legs were mainly machined with 8 to 9 levels of accuracy and the fitting method was gap fit. Due to these manufacturing errors, the two legs of the biped robots were not completely symmetrical. This was particularly evident in reducing the stiffness of the drive chain, because the fit gap directly led to clearance errors in the driving process, which greatly reduced the stiffness of the drive chain. Figure 13 depicts the values of $\varphi_{3}$ with respect to different stiffness during the stance phase. If the right leg had the same stiffness as the left leg, it behaved the same as the left leg (black and red lines). However, when the right leg had a smaller stiffness than the left leg, it performed trajectories that exhibited smaller amplitudes and different wave shapes, although the gait planning for both legs were perfectly symmetrical (the black line and the blue line).

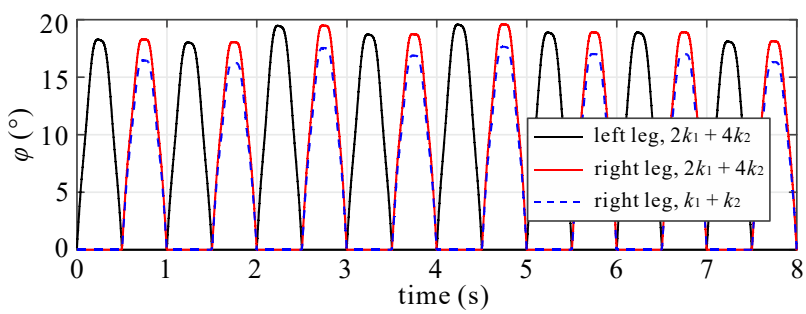

(a)

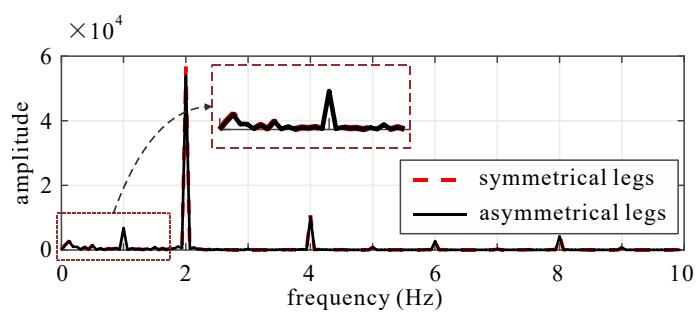

(b)

Figure 13. Simulation results regarding the legs' asymmetry: (a) Values of $\varphi_{3}$ of the two legs with different stiffness (stance phase); (b) FFT analysis of the robot's pitch motion.

As described in Section 2, the difference in the executed execut $\times$ ion of the two legs induced by the stiffness would lead to the upper body vibrating in different directions with different degrees. Figure 13b depicts the FFT analysis of the pitch motion of the robot's body, involving symmetrical or asymmetrical legs. Under the circumstance of symmetrical legs, the peak value occurred at $2.0 \mathrm{~Hz}$, which coincided with the step frequency. However, under the circumstance of asymmetrical legs, a peak value could be observed at $1.0 \mathrm{~Hz}$, which was believed to be caused by the differences in the executed trajectories of the two legs. This demonstrates that greater vibrations of the body would occur if the two legs are not symmetrical. Therefore, manufacturing tolerances should be paid attention during the design and manufacturing process to increase the symmetry of the legs, in particular those that would influence the stiffness of the leg's drive chain. Noting this, currently, AIRO's leg drive chains are manufactured to a class 6-7 accuracy and are fitted with either an overfill fit or with Loctite glue. 


\section{Experiments}

Some experiments were conducted using the biped robot AIRO for validating the influence of the stiffness of the leg's drive chain. Two types of linkages were manufactured with different stiffness, as shown in Figure 14. The black linkages are those used during the preliminary tests and have lower stiffness, while the white linkages are optimized ones with greater stiffness. As shown in Figure 15, using the white linkages, AIRO could alternately lift his legs and touch the ground, successfully achieving omnidirectional walking.

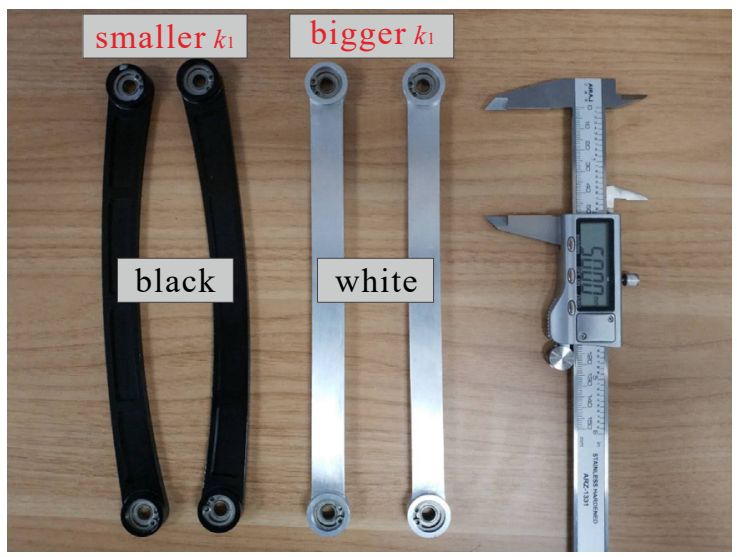

Figure 14. Two types of linkages with different stiffness for experiments.
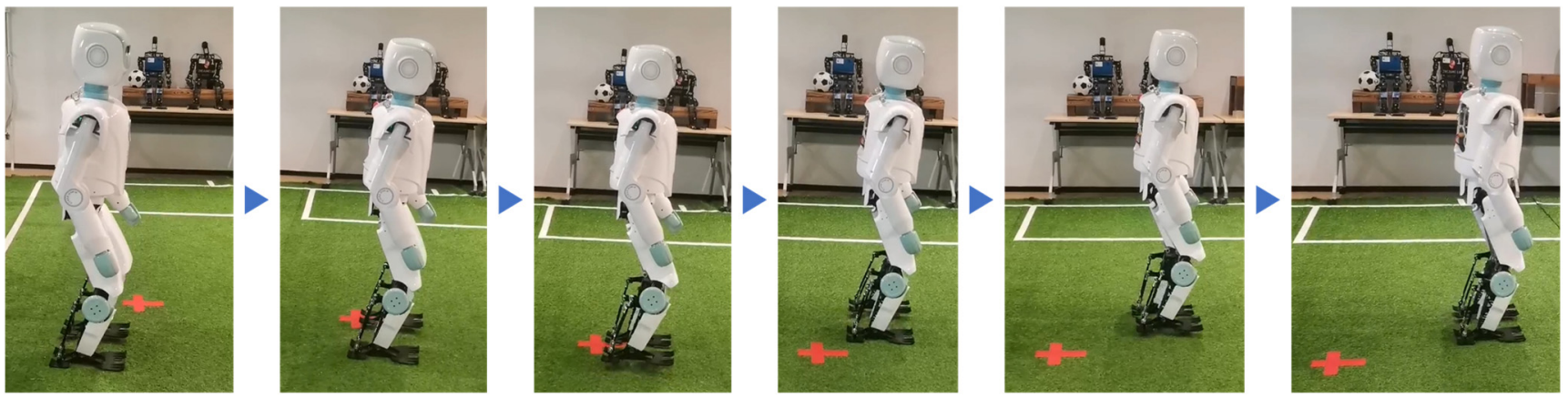

Figure 15. AIRO's omnidirectional Walking with white linkages.

The pitch data of the IMU installed at the hip of the robot was used to analyze the influence of the stiffness of the leg's drive chain, since no encoders were added specifically to measure the execution trajectories of the knee pitch motion. The IMU of AIRO was Xsens MTI-630, which could provide the pitch angle data for analyzing the locomotion dynamics of the robot. Three different cases were analyzed, as depicted in Figure 16: (1) both are black linkages; (2) both are white linkages; (3) one is black; the other is white. During the experiment, the gaiting planning for both legs were completely symmetrical with a step frequency of $2.0 \mathrm{~Hz}$; the rest of the robot was kept constant. The difference in the IMU's pitch angle data could be taken as caused by the linkages.

As depicted in Figure 16, for cases of both black and both white, peaks could be observed around $2.0 \mathrm{~Hz}, 6.0 \mathrm{~Hz}$ and $10.0 \mathrm{~Hz}$. The peaks were larger for white at $2.0 \mathrm{~Hz}$, the same for both cases at $6.0 \mathrm{~Hz}$, and larger for black at $10.0 \mathrm{~Hz}$. As analysis in Section 4 , the peak at $2.0 \mathrm{~Hz}$ indicates the peak-to-peak value, while the high frequencies (here $6.0 \mathrm{~Hz}$ and $10.0 \mathrm{~Hz}$ ) indicate the wave envelope as well as the phase error. This indicates that, for the same gait command, the white linkage obtained a relatively larger peak-to-peak value, a relatively smaller wave envelope, and a smaller phase error, suggesting a better execution of the planned trajectory. The black linkage had a smaller peak-to-peak value, smaller wave envelope, and phase error, suggesting a poorer execution of the planned trajectory. 


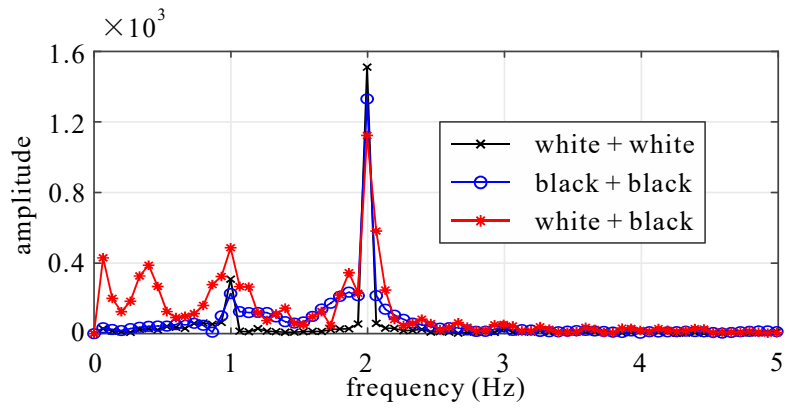

(a)

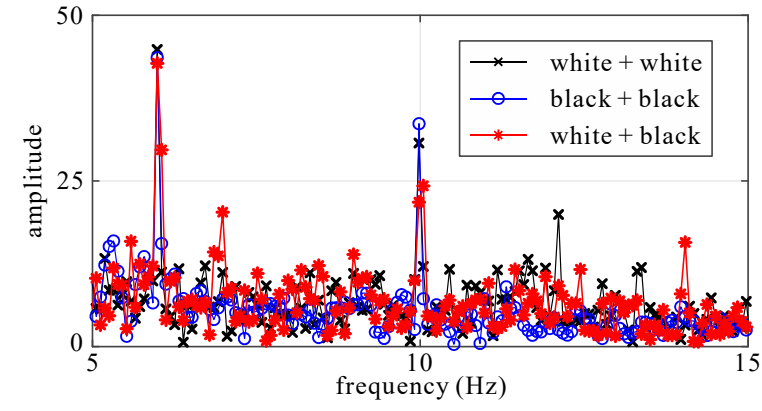

(b)

Figure 16. FFT of the IMU's pitch data: (a) $0-5 \mathrm{~Hz}$; (b) $5-15 \mathrm{~Hz}$.

It is also worth mentioning that the upper body vibrating in different directions with different degrees could, however, still be observed with both white linkages. Moreover, peaks could be observed between 0 and $2.0 \mathrm{~Hz}$, especially at $1.0 \mathrm{~Hz}$. This was mainly due to the asymmetry of the robot's legs. Although the robot was designed to be symmetrical, due to manufacturing and assembly errors, the structure of the legs was not completely identical, which led to a peak at $1.0 \mathrm{~Hz}$. Under this circumstance, the behavior of the left leg stance was repeated only at the next left leg stance, while the behavior of the right leg stance was repeated only at the next right leg stance. Therefore, the frequency was half of the step frequency, i.e., $1.0 \mathrm{~Hz}$.

This phenomenon was particularly evident in the case of one black and one white. Using one black and one white, the stiffness of the two legs was set asymmetry deliberately. The IMU's pitch angle data exhibited a larger peak at $1.0 \mathrm{~Hz}$, and many significant peaks at other frequencies between 0 and $2.0 \mathrm{~Hz}$. These experimental results demonstrate that asymmetry in the stiffness, not the upper body vibrating in many different directions with many different degrees, which could also be observed during the robot walking. It suggests that attention should be paid to the tolerances of the leg structure during the design process, in particular, those that would influence of the drive chain. Reducing the manufacturing assembly errors could improve the symmetry of the legs of biped robots, which would in turn lead to an improvement in the locomotion behavior of biped robots, such as walking smoother with lower vibration of the upper body.

\section{Conclusions and Future Work}

This paper investigates the influence of the stiffness of the leg's drive chain on the locomotion behavior of the biped robots. A mass-spring model was proposed based on the biped robot AIRO built in Zhejiang Lab, and methods for determination of the parameters in the mass-spring model were introduced, including determining the stiffness parameters using ANSYS Workbench and the inertia parameters through dynamic modelling of the biped robot.

Simulation results suggest that special attention should be paid to the stiffness of the drive train of the leg when designing a biped robot to ensure the walking capability of the robot. If the stiffness is not large enough, the motion of the joint will deviate from the gait planning, especially in the stance phase. In addition, manufacturing tolerances should be paid attention for symmetry of the legs of the biped robots. Asymmetry in stiffness of the legs would lead to greater vibration of the robot's body. The simulation analysis was validated via experiments conducted on AIRO.

Upon the analysis of the preliminary testing of AIRO, this paper mainly focused on the knee pitch motion and the related stiffness that would influence the knee pitch motion. As is known, the locomotion of the biped robot is a whole-body dynamic problem and the stiffness of all the joints should be accounted for. Future work will be focused on the influence of the stiffness of the whole body based on the whole-body-control strategy of the biped robot to better understand the biped robot's locomotion behavior. 


\begin{abstract}
Author Contributions: Conceptualization, R.D. and S.S.; Investigation, R.D., S.S., H.Y. and D.N.; Methodology, R.D. and S.S.; Project administration, J.G.; Software, H.Y. and D.N.; Supervision, J.G.; Validation, H.Y. and D.N.; Writing—original draft, R.D. and S.S.; Writing-review and editing, J.G. All authors have read and agreed to the published version of the manuscript.

Funding: This work was supported by NSFC Project (No. 51905495), NSF Project of Zhejiang Province (No. LQ20E050009, No. LQ21F030003, No. LQ22E050024).
\end{abstract}

Conflicts of Interest: The authors declare no conflict of interest.

\title{
References
}

1. Yang, X.; She, H.; Lu, H.; Fukuda, T.; Shen, Y. State of the Art: Bipedal Robots for Lower Limb Rehabilitation. Appl. Sci. 2017, 7, 1182. [CrossRef]

2. Nelson, G.; Saunders, A.; Neville, N.; Swilling, B.; Bondaryk, J.; Billings, D.; Lee, C.; Playter, R.; Raibert, M. Petman: A humanoid robot for testing chemical protective clothing. J. Rob. Soc. Jpn. 2012, 30, 372-377. [CrossRef]

3. Abate, A. Mechanical Design for Robot Locomotion. Ph.D. Thesis, School of Mechanical, Industrial, and Manufacturing Engineering, Oregon State University, Corvallis, OR, USA, 2018.

4. Hirose, M.; Ogawa, K. Honda humanoid robots development. Philos. Trans. A Math. Phys. Eng. Sci. 2007, 365, 11-19. [CrossRef]

5. Tsagarakis, N.G.; Caldwell, D.G.; Negrello, F.; Choi, W.; Baccelliere, L.; Loc, V.G.; Noorden, J.; Muratore, L.; Margan, A.; Cardellino, A.; et al. Walk-man: A high-performance humanoid platform for realistic environments. J. Field Robot. 2017, 34, 1225-1259. [CrossRef]

6. Yuan, H.; Song, S.; Du, R.; Zhao, M.; Zhu, S.; Gu, J. A Capturability-based Control Framework for the Underactuated Bipedal Walking. In Proceedings of the IEEE International Conference on Robotics and Automation (ICRA), Xi'an, China, 30 May-5 June 2021.

7. Park, I.W.; Kim, J.Y.; Lee, J.; Oh, J.H. Mechanical design of the humanoid robot platform, HUBO. Adv. Robot. 2007, 21, 1305-1322. [CrossRef]

8. Hurst, J.W.; Chestnutt, J.E.; Rizzi, A.A. An actuator with physically variable stiffness for highly dynamic legged locomotion. In Proceedings of the IEEE International Conference on Robotics and Automation, New Orleans, LA, USA, 26 April-1 May 2004.

9. Li, X.; Zhou, H.; Zhang, S.; Feng, H.; Fu, Y. WLR-II, a hose-less hydraulic wheel-legged robot. In Proceedings of the 2019 IEEE/RSJ International Conference on Intelligent Robots and Systems (IROS), Macau, China, 4-8 November 2019; pp. $4339-4346$.

10. Zhou, H.; Du, R.; Xie, A.; Yang, H. Theoretical Analysis for the Flow Ripple of a Tandem Crescent Pump with Index Angles. Appl. Sci. 2017, 7, 1148. [CrossRef]

11. Vanderborght, B.; Verrelst, B.; Van Ham, R.; Naudet, J.; Vermeulen, J.; Lefeber, D.; Daerden, F. LUCY, a bipedal walking robot with pneumatic artificial muscles. In Proceedings of the IEEE Conference on Mechatronics and Robotics, Singapore, 1-3 December 2004; pp. 106-114.

12. Ahmad Sharbafi, M.; Shin, H.; Zhao, G.; Hosoda, K.; Seyfarth, A. Electric-Pneumatic Actuator: A New Muscle for Locomotion. Actuators 2017, 6, 30. [CrossRef]

13. Song, S.; Tang, C.; Wang, Z.; Yan, G. Active disturbance rejection controller design for stable walking of a compass-like biped. Trans. Institute Meas. Control 2018, 40, 4063-4077. [CrossRef]

14. Yuan, H.; Ge, Y.; Gan, C. Control strategy for gait transition of an underactuated 3D bipedal robot. Front. Inform. Technol. Electron. Eng. 2019, 20, 1026-1035. [CrossRef]

15. Gong, Y.; Hartley, R.; Da, X.; Hereid, A.; Harib, O.; Huang, J.K.; Grizzle, J. Feedback control of a cassie bipedal robot: Walking, standing, and riding a segway. In Proceedings of the 2019 American Control Conference (ACC), Philadelphia, PA, USA, 10-12 July 2019.

16. Carbone, G.; Lim, H.; Takanishi, A.; Ceccarelli, M. Stiffness analysis of biped humanoid robot WABIAN-RIV. Mech. Mach. Theory 2006, 41, 17-40. [CrossRef]

17. Carbone, G.; Lim, H.; Takanishi, A.; Ceccarelli, M. Stiffness analysis of the humanoid robot WABIAN-RIV: Modelling. In Proceedings of the 2003 IEEE International Conference on Robotics and Automation (ICRA), Taiwan, China, 14-19 September 2003.

18. Carbone, G.; Lim, H.; Takanishi, A.; Ceccarelli, M. Numerical and experimental estimation of stiffness performances for the humanoid robot WABIAN-RV. In Proceedings of the 2003 IEEE/ASME International Conference on Advanced Intelligent Mechatronics (AIM), Kobe, Japan, 20-24 July 2003.

19. Kwon, W.; Kim, H.; Park, J.; Roh, C.H.; Lee, J.; Park, J.; Kim, W.K.; Roh, K. Biped humanoid robot Mahru III. In Proceedings of the 2007 7th IEEE-RAS International Conference on Humanoid Robots, Pittsburgh, PA, USA, 29 November-1 December 2007.

20. Kim, J.; Lee, Y.; Kwon, S.; Seo, K.; Roh, K. Development of the lower limbs for a humanoid robot. In Proceedings of the 2012 IEEE/RSJ International Conference on Intelligent Robots and Systems, Algarve, Portugal, 7-12 October 2012.

21. Lohmeier, S. Design and Realization of a Humanoid Robot for Fast and Autonomous Bipedal Locomotion. Ph.D. Thesis, Technische Universität München, München, Germany, 2010.

22. Lohmeier, S.; Thomas, B.; Heinz, U. Humanoid robot LOLA. In Proceedings of the 2009 IEEE International Conference on Robotics and Automation (ICRA), Kobe, Japan, 12-17 May 2009.

23. Lohmeier, S.; Buschmann, T.; Schwienbacher, M.; Ulbrich, H.; Pfeiffer, F. Leg design for a humanoid walking robot. In Proceedings of the 2006 6th IEEE-RAS International Conference on Humanoid Robots, Genova, Italy, 4-6 December 2006. 
24. Xiong, X.; Ames, A.D. Bipedal hopping: Reduced-order model embedding via optimization-based control. In Proceedings of the 2018 IEEE/RSJ International Conference on Intelligent Robots and Systems (IROS), Madrid, Spain, 1-5 October 2018.

25. Xiong, X.; Ames, A.D. Coupling reduced order models via feedback control for $3 \mathrm{~d}$ underactuated bipedal robotic walking. In Proceedings of the 2018 IEEE-RAS 18th International Conference on Humanoid Robots (Humanoids), Beijing, China, 6-9 November 2018

26. De Luca, A.; Book, W. Robots with Flexible Elements. In Springer Handbook of Robotics; Springer: Cham, Switzerland, 2016; pp. 243-282.

27. Ma, Z.; Zuom, S.; Chen, B.; Liu, J. Friction prediction and validation of a variable stiffness lower limb exosuit based on finite element analysis. Actuators 2021, 10, 151. [CrossRef]

28. Palli, G.; Melchiorri, C.; De Luca, A. On the feedback linearization of robots with variable joint stiffness. In Proceedings of the 2008 IEEE International Conference on Robotics and Automation (ICRA), Pasadena, CA, USA, 19-23 May 2008.

29. Klimchik, A.; Caro, S.; Furet, B.; Pashkevich, A. Complete stiffness model for a serial robot. In Proceedings of the 2014 11th International Conference on Informatics in Control, Automation and Robotics (ICINCO), Vienna, Austria, 2-4 September 2014.

30. Pashkevich, A.; Klimchik, A.; Chablat, D. Enhanced stiffness modeling of manipulators with passive joints. Mech. Mach. Theory 2011, 46, 662-679. [CrossRef]

31. Kim, M.; Park, J.; Kim, J.; Kim, M.; Lee, D. Stiffness decomposition and design optimization of under-actuated tendon-driven robotic systems. In Proceedings of the 2018 IEEE International Conference on Robotics and Automation (ICRA), Brisbane, Australia, 21-25 May 2018.

32. Geeroms, J.; Flynn, L.; Jimenez-Fabian, R.; Vanderborght, B.; Lefeber, B. Design and energetic evaluation of a prosthetic knee joint actuator with a lockable parallel spring. Bioinspir. Biomim. 2017, 12, 026002. [CrossRef] [PubMed] 